\title{
PAPERS \\ OF THE \\ BRITISH SCHOOL AT ROME
}




\title{
PAPERS OF THE BRITISH SCHOOL AT ROME
}

\author{
VOLUME XLVI
}

$197^{8}$

PLBLISHED BY THE

BRITISH SCHOOL AT ROME AT I, LOWTHER GARIDENS, EXHIBITION ROAD, I.ONDON, SW7 $2 \mathrm{AA}$ 
(C) Copyright The British School at Rome, I, Lowther Gardens, London $S_{7} W_{2 A A}$

$I S S \mathcal{N} 0068-2462$

Printed in Great Britain

BY STEPHEN AUSTIN AND SONS LIMITED

Hertford, England 
The Papers of The British School at Rome exist to publish work relating to the archaeology, history and literature of Italy and other parts of the Mediterranean area up to early modern times, in the first instance by the staff of the School and by its present and former members. The Papers are edited by the Publications Committee of the Faculty of Archaeology, History and Letters of the British School.

The present members of the Committee are:

Dr. G. W. Barker

Dr. D. Chambers

Mr. Michael H. Crawford (Chairman)

Dr. Richard Duncan-Jones

Mr. M. W. Frederiksen

Professor W. V. Harris (University of Columbia)

Dr. D. B. Whitehouse, Director of the School (ex-officio)

Professor J. J. Wilkes

Dr. P. A. Bulloch (Editorial Secretary)

Intending contributors are advised to get in touch with Dr. P. A. Bulloch, c/o The British School at Rome, I Lowther Gardens, Exhibition Road, London SW7 2AA, before preparing their MSS in final form, since they will be required to conform to the conventions currently followed in the Papers.

The Faculty also publishes a series of Supplementary Volumes, to accommodate works on a scale larger than is suitable for the Papers.

The present volume of the Papers is available at a cost of $\mathcal{E}$ io to individual subscribers to the Faculty, $E_{12}$ to others ( $\mathscr{1}_{1} 4$ overseas), postage and packing extra.

A full List of Publications available is shewn overleaf. 


\section{LIST OF PUBLICATIONS}

Orders and enquiries should be sent to Mrs. K. M. Stedman, Secretary, The British School at Rome, I Low ther Gardens, Exhibition Road, London SW7 2AA. Cheques should be made payable to "The British Sichool at Rome".

\section{The Papers of The British School at Rome}

Volumes i to xxiv inclusive have been reproduced by the Johnson Reprint Corporation and orders should be sent to: Johnson Reprint Corporation, Department S.L.., I I Fifth Avenue, New York, N.Y. rooo', L.S.A.

Volumes xxv (New series xii) I957 to xlv (New series xxxii) 1977 are available at the

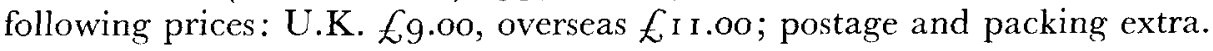

Late Roman Pottery, by J. W. Hayes, is being re-issued with a substantial Supplement, which is also available separately.

\section{Supplementary Publications}

Porta Argentariorum

D. E. L. HAYNES and

P. E. D. HIRST, 1939

The Papal State under Martin V

PETER PARTNER, $195^{8}$

Late Roman Pottery

J. W. HAYES, 1972

(with Supplement, I979)

Medieval Malta: Studies on Malta before the Knights

Ed. A. T. LUTTRELL, I975

Approaches to Medieval Malta

A. T. LU'T'TRELL, I 975

Reprinted from Medieval Malta:

Studies on Malta before the Knights,

ed. A. T. Luttrell, 1975)

A Faliscan Town in South Etruria:

Excavations at Narce 1966-7 I

Ed. T. W. POTTER, 1976

Supplement to Late Roman Pottery

J. W. HAYES, I 979

The Late Republican Villa at Posto, Francolise M. AYLWIN COTTON, I979
Price, postage and packing extra

L.K. Overseas

$2.3 .5^{\circ} \quad £ .5 \cdot 5^{\circ}$

$264 \quad-\quad £ 5.5^{\circ} \quad £ 7.5^{\circ}$

477

c. $3^{2}$

$23^{2}$

24

E. 3.50

EI $5 \cdot 5^{\circ}$

$\left.{ }_{-}^{23}\right\}$

$£ 22.00 \quad \& 24.00$

70 $\quad$ - Li.jo $\quad$ L3.00 


\section{Offprints from the Papers of The British School at Rome}

From Volume $\mathrm{xl}$ (New series xxvii) 1972 onwards, selected offprints are available as classified below.

\section{Prehistoric}

An Apennine Bronze Age Settlement at Petrella, Molise GRAEME BARKER, xliv, 1976
Price, postage and packing included

8 op

\section{Roman}

Stoicism and the Principate

$$
29
$$

E.1.10

The Ancient Roads of Southeastern Lucania 20

R. J. BUCK, xliii, I 975

A Bibliography of Professor Donald Strong 6 xliii, 1975

The Cassia-Clodia Survey

P. HEMPHILL, xliii, 1975

2

$£ 2.30$

Architecture and Sculpture: The Activities

of the Cossutii

E: RAWSON, xliii, 1975

The Earliest. Wall Mosaics in Italy

F. SEAR, xliii, 1975

Jobs in the Household of Livia

SUSAN TREGGIARI, xliii, I97j

Economy and Society of Mediolanum under the Principate

P. D. A. GARNSEY, xliv, 1976

Conservatism and Change in Roman

Religion

8op

5op

J. A. NORTH, xliv, 1976

I2

15

3

8op

I 2

$3^{\circ}$

เ6

6 op

fi.10

6 op

6 op 
Gravina-di-Puglia III

Pages Plates

JOAN DU PLAT TAYLOR and others,

(Part One), xliv, 1976

(Part Two), xlv, I977

Public Building in Rome between the Second Punic War and Sulla

F. COARELLI, xlv, I 977

Stucco Decorations at Baia

ROGER LING, xlv, 1977

A Roman Villa near Anguillara Sabazia

M. LYTTELTON and

F. SEAR, xlv, 1977

In praedis Iuliae Felicis

HELEN WHITFHOUSE, xlv, 1977
Price, postage and

packing included $\left.\begin{array}{rr}85 & 10 \\ 69 & 7\end{array}\right\} \quad \notin 6.00$

23

3

$£$ I.10

28

13

$E^{1} \cdot 5^{\circ}$

25

12

$£$ I.50

I6

4

8op

\section{Medieval}

Excavations at Tuscania, I 973

J. B. WARD-PERKINS and others, xli, I 973

An Umbrian Abbey: San Paolo di Valdiponte, 49 Part One

A. T. LUTTRELL, $x l, 197^{2}$

Ceramica Laziale

D. B. WHITEHOUSE, xliv, 1976 


\section{CONTENTS}

Glaude Nicolet, Le Stipendium des Alliés Italiens avant la Guerre Sociale... 1

Elizabeth Rawson, The Introduction of Logical Organisation in Roman Prose Literature

Graeme Barker, John Lloyd, Derrick Webley, A Classical Landscape in Molise

Deane R. Blackman, The Volume of Water delivered by the Four Great Aqueducts of Rome.

A. C. G. Smith, The Date of the 'Grandi Terme' of Hadrian's Villa at Tivoli 73

Walter Widrig, Two Churches at Latrun in Gyrenaica.

94

C. J. Wiскнам, Historical and Topographical Notes on Early Mediaeval South Etruria (Part One) 


\section{SOMMARII}

\section{LO STIPENDIO DEGLI ALLEATI ITALIANI PRIMA DELLA GUERRA SOCIALE}

L'affermazione secondo la quale i Romani, a differenza, ad esempio, degli Ateniesi, abbiano preteso dai loro alleati italiani solo contributi in uomini per le loro operazioni militari escludendo contributi in denaro è inesatto. Testi espliciti di Polibio e Tito Livio, mostrano al contrario che gli alleati dovevano su richiesta dei Romani, fornire in una sola volta contingenti (in virtù della formula togatorum) e denaro per stipendiarli ed equipaggiarli. Questo stipendio proporzionale ai contingenti è reso permanente e proporzionale al census per le dodici colonie che si ribellarono nel 209 e furono punite nel 204. I soldati alleati non subivano trattenute sul vitto ed equipaggiamento (al contrario dei soldati romani); si può anche dire che il peso delle città alleate era leggermente più pesante di quello del Tesoro Romano. Questa situazione è durata anche dopo il 167, data in cui, come è noto, il tributo romano è stato soppresso essendo reso inutile dalle risorse propric del Tesoro. Un testo di Appiano, due brani della lex repetundarum epigrafica e un brano delle Orazioni contro Verre mostrano molto chiaramente che, nel periodo antecedente alla guerra sociale, le prestazioni in denaro erano sempre pretese dagli alleati italiani. Quest'ultimi hanno dunque contribuito sempre più pesantemente alle spese della conquista romana, essendo in questo periodo privati dei vantaggi diretti o indiretti che questa stessa conquista procurava ai cittadini romani. Questo è certamente un fatto che contribuisce a spiegare la guerra sociale.

Gitaude Nicolet

\section{L'INTRODUZIONE DELL'ORGANIZZAZIONE LOGICA NELLA LETTERATURA DI PROSA ROMANA}

Fu dall'inizio del primo secolo a.C. che i Romani impararono a formulare dissertazioni su tutti gli argomenti (c non semplicemente testi elementari) in conformità alle tecniche di definizione e divisione, in definitiva derivate dalla dialettica greca, sebbene essi le applichino con estrema semplicità ed entusiasmo. Il ruolo di queste tecniche è considerato, tra gli altri, nei lavori di Varro e Cicero; come la loro applicazione nei discussi argomenti di giurisprudenza. Alcuni argomenti, o parte degli argomenti è ancora da organizzare in questo modo nel periodo imperiale, sebbene il metodo fosse considerato più che sicuro. L'intero sviluppo fu di grande importanza per la storia intellettuale, al pari del modo in cui i Romani appresero dai Greci anche ad organizzare la narrativa o un discorso in una esposizione chiara e comprensibile.

ELISABETH RAWSON

\section{UN PAESAGGIO CLASSICO IN MOLISE}

La relazione discute sui modelli di sistemazione classica scoperti da recenti ed intensivi studi nella Valle Biferno nel Molise. Questo lavoro è parte di un'indagine pluriennale della sistemazione storica della valle, effettuato da archeologi storici e studiosi di scienze naturali. Due zone della valle inferiore sono illustrati come i nostri esempi (Fig. 2 e 3). In entrambe le zone un primo esempio di numerose piccole fattorie fu sostituito, nei recenti tempi Repubblicani, da un sistema di pochissime fattorie, ma molto più grandi (ville?), specialmente lontano dai maggiori centri urbani. Noi discutiamo tre modelli possibili per spiegare questa trasformazione in sistemazione: (1) pastorizia specializzata, o (2) produzione arabile aumentata, (entrambi controllate da ricche famiglie in citta come Larino), o (3) nuclei di sistemazione senza cambiamenti nell'uso della terra. Noi presentiamo le ragioni per cui crediamo che il primo modello debba essere preferito per il presente. 


\section{IL VOLUME D'ACQUA DISTRIBUITA DAI QUATTRO GRANDI ACQUEDOTTI DI ROMA}

Questo studio si propone, attraverso calcoli idraulici, di stabilire il volume d'acqua distribuita dai quattro grandi acquedotti della Roma antica. L'essenza dell'argomento è la profondità dell'acqua in un canale aperto che cambia la sua larghezza e la cui pendenza varia in maniera complessa ma prevedibile. Dal momento che l'ampiezza e la pendenza di alcuni punti degli acquedotti romani sembrano essere sufficientemente ben definite per permettre calcoli di questo tipo, ed inoltre in alcuni di questi casi anche l'altezza del canale è accessibile, un limite superiore può essere posto sullo scarico. Il risultato numerico più importante è che la distribuzione di questi quattro acquedotti era probabilmente di $7 \mathrm{~m}^{3}$ al secondo (600 $000 \mathrm{~m}$ cubi al giorno).

Deane R. Blackman

\section{LA DATA DELLE 'GRANDI TERME’ DELLA VILLA ADRIANA A TIVOLI}

Nel suo studio sui bolli laterizi di Villa Adriana, Bloch attribuì le grandi terme ad un edificio della terza fase edilizia, nonostante essi contengano molti bolli non trovabili altrove in edifici posteriori alla prima fase della Villa e del Pantheon (il quale, pace W. D. Heilmeyer, è tuttora da attribuire ai primi anni del regno di Adriano).

La data più recente di Bloch per le terme dipende dall'uso della formula 'Lucilla Veri' sul bollo CIL XV 1049, dal momento che secondo Mommsen e Dressel questa formula era usata solo sui bolli post-Adrianei; qui si è sostenuto che il resto del testo di questo bollo é più caratteristico del primo periodo Adrianeo di quanto suggerisca il suo contesto, e perciò dato che né i resti dei bolli laterizi nelle terme né considerazioni topografiche presentano seri ostacoli, le grandi terme dovrebbero essere riattribuite alla prima fase di Bloch.

Senza le grandi terme la terza fase di Bloch è di scarsa importanza, dato che gli altri edifici attribuiti a detta data potrebbero benissimo appartenere alla seconda fase. Probabilmente, quindi, non ci fu alcun periodo a sé stante di una maggiore attività edilizia alla Villa durante gli ultimi anni di Adriano in Italia.

A. C. G. S Sitth

\section{DUE CHIESE A LATRUN IN CIRENAICA}

Questo articolo tratta di uno scavo di due chiese basilicali del paese di Latrun sulla costa della Girenaica, Libia. Latrun è identificato come 1' 'Erythron' del $2^{\circ}$ secolo d.C. menzionato dal geografo Tolomeo e come la sede di un vescovo da almeno l'inizio del $5^{\circ}$ secolo. Le due chiese quasi identiche nella forma, sono insolite in Cirenaica per l'elevazione delle loro gallerie e per materiali d'importazione quali le basi di marmo delle colonne, fusti, capitelli e blocchi di imposte. La forma è derivata da un prototipo di Costantinopoli del $5^{0}$ secolo ed è solo leggermente modificata per rispondere alle esigenze locali fino alla trasformazione della chiesa più grande, la cattedrale propriamente detta, in un castrum. Le chiese più piccole contenevano tombe disturbate e senza dubbio la tomba di un martire. La data proposta per le chiese è il 500 sulla base di una datazione accettata per il tipo di pianta, lo sviluppo generale delle strutture delle chiese in Cirenaica, il carattere stilistico dei rivestimenti di marmo, e la storia politica e religiosa della regione. Inoltre l'autore specula sulla possibilità di mecenatismo imperiale, partecipazione nella controversia tra Ortodossi e Monofisiti e l'esistenza del culto di un martire di grande importanza a Latrun.

Walter Widrig 


\section{NOTE STORICHE E TOPOGRAFICHE SULL'ETRURIA MERIDIONALE DEL PRIMO MEDIOEVO: PARTE I}

Questo articolo forma la prima parte di una duplice indagine di documenti del periodo 700-1200, con lo scopo di sviluppare una documentazione medievale controparte alle indagini della BSR sull'Etruria Meridionale, per una regione comprendente la zona di indagine di Ager Veientanus le cui estensioni si allungano verso ovest a Cesano e verso est al Tevere. La regione è più o meno inclusa in una lista di chiese del $11^{\circ}$ secolo, lista che sopravvive in una copia nel registro di Papa Gregorio IX, ci dà una base per l'analisi documentaria di esempi di insediamenti medievali nel $11^{\circ}$ secolo. In questo articolo le zone intorno all'Isola Farnese, Cesano, Fanello, Sacrofano e le pianure a est dell'Isola sono analizzate. Nel $11^{0}$ secolo i castelli della pianura sembrano essere stati luoghi per sistemazioni in nuclei, ma lungo le strade consolari e negli altopiani gli insediamenti sparsi sopravvissero. Per la maggior parte, la proprietà in quel periodo era molto frammentaria e ciò sembra essere stato per $1^{\prime} 8^{\circ}$ e $9^{0}$ secolo, periodo 'domusculta', che l'autore non considera come un periodo di bonifica e consolidamento. Conclusioni generali appariranno nella II parte.

G. J. WickHam 